NOTA CIENTÍFICA

\title{
Algunos acantocéfalos de la familia Oligacanthorhynchidae del Perú
}

\section{Some acanthocephalans of the family Oligacanthorhynchidae from Peru}

\section{Luis A. Gomez-Puerta}

Laboratorio de Medicina Veterinaria Preventiva. Facultad de Medicina Veterinaria. Universidad Nacional Mayor de San Marcos. Av. Circunvalación 2800, San Borja. Lima, Perú.

E-mail: lucho92@yahoo.com

$\begin{array}{ll}\text { Presentado: } & 28 / 05 / 2011 \\ \text { Aceptado: } & 17 / 10 / 2011\end{array}$ $\begin{array}{ll}\text { Aceptado: } & 17 / 10 / 2011 \\ \text { Publicado online: } & 08 / 02 / 2012\end{array}$

\section{Resumen}

Cuatro especies de acantocéfalos pertenecientes a la familia Oligacanthorhynchidae son estudiados. Dos de ellas corresponden a nuevos hallazgos geográficos. Adicionalmente se registra por primera vez en el Perú a Oligacanthorhynchus carinii y Oligacanthorhynchus major.

Palabras clave: Acantocefalos, Oligacanthorhynchidae, Perú.

\section{Abstract}

Four species of acanthocephalan (Oligacanthorhynchidae) were studied. Two species are new geographical records. Additionally, Oligacanthorhynchus carinii and Oligacanthorhynchus major are registered for the first time in Peru.

Keywords: Acanthocephala, Oligacanthorhynchidae, Perú.

\section{Introducción}

Los acantocéfalos son un grupo de parásitos intestinales que infectan una variedad de vertebrados. Presentan un ciclo de vida indirecto, necesitando de un invertebrado como hospedador intermediario, en ellos se desarrollara la forma larvaria denominada cystacantho (Petrochenko 1956).

Los estudios sobre acantocéfalos en el Perú son limitados. La gran mayoría de especies conocidas hasta ahora han sido registradas en peces y aves silvestres (Tantaleán et al. 2005). En el presente trabajo se dan a conocer nuevos acantocéfalos para el Perú, así como nuevos hallazgos geográficos para algunas especies ya registradas en estudios anteriores.

\section{Material y métodos}

Los acantocéfalos se colectaron directamente de los hospederos y fueron relajados en agua destilada hasta que la proboscis estuvo completamente evertida. Luego, los parásitos se fijaron y preservaron en etanol al 70\%. Para el estudio morfológico, los acantocéfalos fueron aclarados en una solución de alcoholfenol (1:2 V/V) y coloreados con carmín de Semichon. Algunos

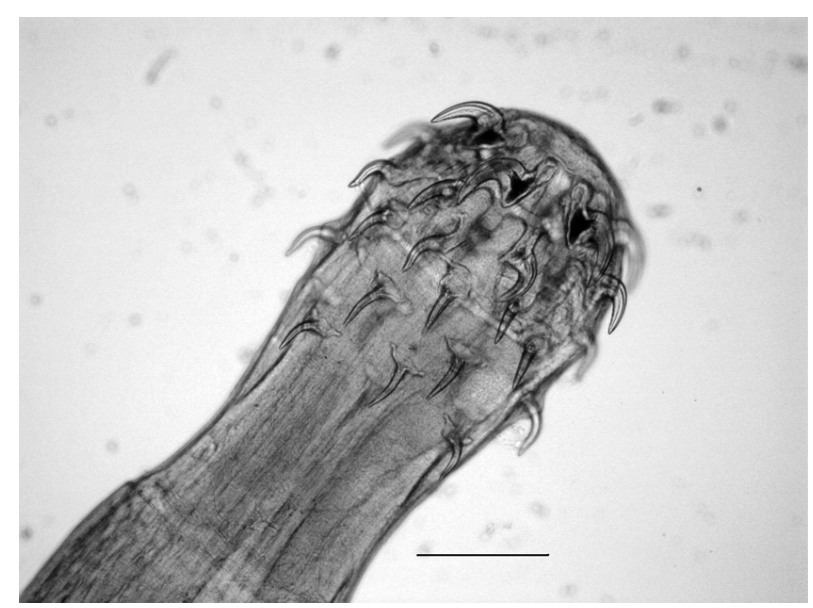

Figura 1. Oligacanthorhynchus carinii. Probóscide de un espécimen adulto. Escala 0,2 $\mathrm{mm}$ parásitos fueron disectados para el estudio anatómico de sus órganos genitales. Las medidas y fotografías se realizaron usando un microscopio Carl Zeiss Axioskiop-40.

Para la identificación de los especímenes se utilizaron las claves propuestas por Travassos (1917), Petrochenko (1956) y Richardson y Barger (2006). La nomenclatura taxonómica sigue a Amin (1985). Parte de las muestras examinadas se encuentran depositadas en la Colección Helmintológica y de Invertebrados Relacionados del Museo de Historia Natural de la UNMSM (MUSM) Lima, Perú.

\section{Resultados}

FAMILIA OLIGACANTHORHYNCHIDAE OUTHWELL \& MACFIE, 1925

\section{Oligacanthorhynchus carinii (Travassos, 1917) Schmidt, 1972}

(Fig. 1)

Hospedero: Dasypus novemcinctus (Dasypopidae).

Localización: Intestino delgado.

Localidad: Iquitos ( $\left.3^{\circ} 45^{\prime} 00^{\prime \prime} S, 73^{\circ} 15^{\prime} 00^{\prime \prime} \mathrm{W}\right)$, Loreto

Deposito No: MUSM 3016

Comentario: Oligacanthorhynchus carinii mide de 140 a 220 $\mathrm{mm}$ de longitud por 2 a 2,5 mm de ancho. Fue descrito por vez primera en el Brasil por Travassos (1917) como Hamanniella carinii; posteriormente, Meyer (1932 o 1933? revisar en bibliografia) consideró la especie dentro del género Travassosia. Schmidt (1972) realizó una revisión de la familia Oligacanthorhynchidae y consideró a los géneros Hamaniella y Travassosia como sinónimos del genero Oligacanthorhynchus.

Esta especie ha sido descrita en mamíferos dasipódidos (Dasypodidae): Dasypus novemcinctus de Brasil (Travassos, 1917; Lent y Freitas, 1938); en Tolypeutus tricinctus conurus de Bolivia (Meyer, 1933); en Chaetophractus vellerosus y Tolypeutus mataco procedentes de Argentina (Martínez, 1984) y en Tolypeutes mata- 


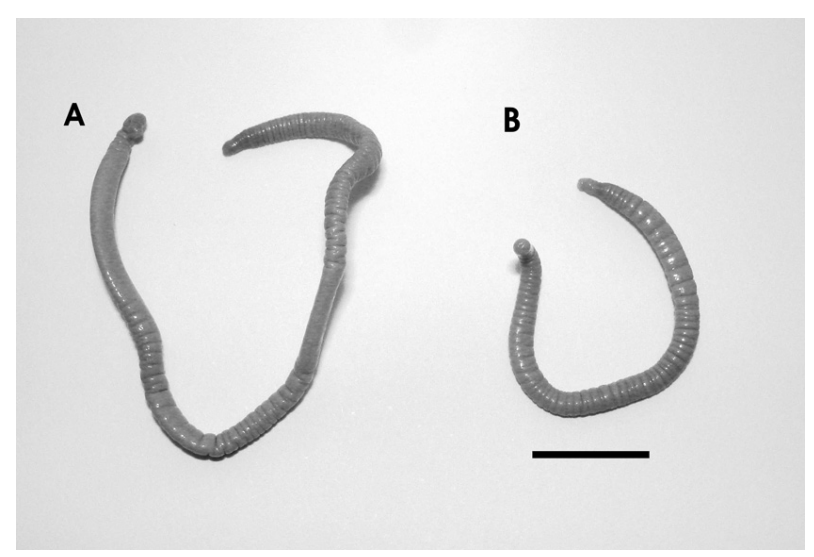

Figura 2. Oligacanthorhynchus major. (A) Macho adulto. (B) Macho inmaduro. Escala 1,0 cm.

cus de Paraguay. Con nuestro registro, ampliamos la distribución geográfica de esta especie de acantocéfalo.

\section{Oligacanthorhynchus major (Machado-Filho, 1963) Schmidt, 1972}

(Fig. 2)

Hospedero: Tayassu pecari (Tayassuidae)

Localización: Intestino delgado.

Localidad: Tambopata (12³5’36”S, 69¹0’35”W), Madre de Dios.

\section{Deposito No: MUSM 3017}

Comentario: Acantocéfalos relativamente grandes, los machos pueden llegar a medir hasta $498 \mathrm{~mm}$ y las hembras hasta 697 mm. Machado-Filho (1963) describe a Macracanthorhynchus major parasitando el intestino delgado del pecarí de collar (Tayassu tajacu) proveniente de Brasil. Posteriormente, Schmidt (1972) transfiere esta especie de acantocéfalo dentro del género Oligacanthorhynchus. La especie $O$. major ha sido descrita parasitando el intestino delgado de pecaríes (Tayassu pecari y $T$. tajacu) provenientes de Brasil y Bolivia. Este hallazgo representa el primero registro de $O$. major para el Perú.

\section{Macracanthorhynchus hirudinaceus (Pallas, 1781)}

Hospedero: Sus scrofa f. domestica (Suidae)

Localización: Intestino delgado.

Localidad: Capitán Hoyle (0405’00”S, 8053’00”W), Tumbes.

\section{Deposito No: MUSM 3018}

Comentario: $M$. hirudinaceus es un parasito del intestino delgado del cerdo que presenta una distribución cosmopolita. Actualmente en el Perú, se conoce su distribución en las localidades de: Cajamarca, Cajabamba, Celendín, Hualgayoc, San Marcos, San Pablo (Cajamarca); Huanuco, Leoncio Prado (Huánuco); Chiclayo, Ferreñafe (Lambayeque); Pucallpa (Ucayali); con este hallazgo ampliamos su distribución en el Perú.

Las infecciones en los cerdos causan diversos cuadros patológicos. Las infestaciones medias por $M$. hirudinaceus no son muy peligrosas, pero las infestaciones intensas pueden provocar retraso en el crecimiento y emaciación, así como cuadros de peritonitis ocasionada por la perforación de la pared intestinal por parte del parasito. En lo que respecta a Salud Publica, se han reportado casos humanos de acantocefalosis por $M$. hirudinaceus en diversas partes del mundo (Radomyos et al. 1989, Barnish y Misch 1987, Hemsrichart et al. 1983, Leng et al. 1983).

\section{Prosthenorchis elegans (Diesing, 1851)}

\author{
Hospedero: Saimiri sciureus (Cebidae) \\ Localización: Intestino delgado. \\ Localidad: Lima.
}

Deposito No: MUSM 3019

Comentario: P. elegans es un parasito común de primates del nuevo mundo. Fue reportado por primera vez en el Perú en el primate Saimiri sciureus (Dunn, 1963). Posteriores estudios ampliaron la lista de hospederos definitivos, registrándose hasta ahora en Saguinus labiatus, S. mystax, Saimiri boliviensis y $S$. sciureus provenientes de Loreto (Michaud et al., 2003); y en $S$. sciureus de la reserva Tambopata en Madre de Dios (Phillips et al. 2004). En el presente estudio el acantocéfalo fue colectado de un ejemplar de $S$. sciureus criado como mascota en la ciudad de Lima.

Este acantocéfalo se propaga fácilmente en colonias de monos en cautiverio, utilizando a las cucarachas o a algunos escarabajos como hospederos intermediarios (Stunkard 1965). La acantocefalosis ocasionada por $P$. elegans presenta dos síndromes: un síndrome crónico y uno agudo. El curso crónico se caracteriza por una diarrea acuosa por varios meses, con cuadros de anorexia y caquexia. El curso agudo dura menos de un día produciendo la muerte del animal a causa de una peritonitis bacteriana por perforación de la pared intestinal por la proboscis del parasito.

Los registros sobre acantocéfalos en el Perú son principalmente por hallazgos accidentales en las necropsias de animales, tales como en el presente trabajo. El registro por primera vez en el Perú de los acantocéfalos $O$. carinii y $O$. major puede deberse a la escasez de estudios parasitológicos en mamíferos dasipódidos y tayasuidos peruanos.

Con los nuevos registros de los acantocéfalos $O$. carinii y $O$. major, el estatus de las familia Oligacanthorhynchidae en el Perú pasaría a conformarse por seis especies incluyendo a $M$. hirudinaceus, P. elegans, Oncicola spirula y Oncicola canis.

\section{Literatura citada}

Amin O.M. 1985. Classification. In: D. W. T. Crompton and B. B. Nickol, eds. Biology of the Acanthocephala. Cambridge University Press, Cambridge, U.K. Pages 27-72

Barnish G. \& K.A. Misch. 1987. Unusual cases of parasitic infections in Papua New Guinea. Am. J. Trop. Med. Hyg. 37:585-587.

Dunn F.L. 1963. Acanthocephalans and cestodes of South American monkeys and marmosets. J. Parasitol, v. 47, p. $717-722$.

Hemsrichart V., C. Pichyangkura, S. Chitchang \& U. Vutichamnong. 1983. Eosinophilic enteritis due to Macracanthorhynchus hirudinaceus infection: report of 3 cases. J. Med. Assoc. Thai. 66: 303-310. 
Leng Y.J., W.D. Huang \& P.N. Liang. 1983. Human infection with Macracanthorhynchus hirudinaceus Travassos, 1916 in Guangdong Province, with notes on its prevalence in China. Ann. Trop. Med. Parasitol. 77: 107-109.

Lent H. \& Freitas J.F. 1938. Pesquisas helmintologicas realisadas no Estado do Para. VI Acanthocephala. Mem. Inst. Oswaldo Cruz. 33: 455

Machado-Filho D.A. 1963. Nova espécie do gênero "Macracanthorhynchus" Travassos, 1916, parasito de "Tayassu Tajacu" (L., 1758) (Archiacanthocephala, Oligacanthorhynchidae). Rev. Bras. Biol. 23: 409-412.

Martínez F.A. 1984. Hamanniella carinii Travassos, 1916 (Acanthocephalo; Gigantorhynchidae) en Dasipodideos de Argentina. Bol. Chil. Parasitol. 39: 37-38.

Meyer A. 1933. Acanthocephala. Bronn's Klassen und Ordnungen des Tierreichs Vol. $4(11,2)$.

Michaud C., M. Tantalean, C. Ique, E. Montoya \& A. Gozalo. 2003. A survey for helminth parasites in feral New World non-human primate populations and its comparison with parasitological data from man in the región. J. Med. Primatol. 32: 341-345

Petrochenko V.I. 1956. Acanthocephala of domestic and wild animals. Izdatelstvo Akademii Nauk SSSR, Moscow, Russia, 465 p.
Phillips K.A., M.E. Haas, B.W. Grafton \& M. Yrivarren. 2004. Survey of the gastrointestinal parasites of the primate community at Tambopata National Reserve, Peru. J. Zool. Lond. 264, 149-151

Radomyos P., A. Chobchuanchom \& A. Tungtrongchitr. 1989. Intestinal perforation due to Macracanthorhynchus hirudinaceus infection in Thailand. Trop. Med. Parasitol. 40: 476-477.

Richardson D.J. \& M.A. Barger. 2006. Redescription of Oligacanthorhynchus major (Machado-Filho, 1963) Schmidt, 1972 (Acanthocephala: Oligacanthorhynchidae) from the white-lipped peccary (Tayassu pecari) in Bolivia. Comp. Parasitol. 73: 157-160.

Schmidt G.D. 1972. Revision of the class Archiacanthocephala Meyer, 1931 (Phylum Acanthocephala), with emphasis on Oligacanthorhynchidae Southwell et Macfie, 1925. J. Parasitol. 58: 290-297.

Stunkard H.W. 1965. New intermediate hosts in the life cycle of Prosthenorchis elegans (Diesing, 1851), an acanthocephalan parasite of primates. J. Parasitol. 51: 645-649.

Tantaleán M., L. Sanchez, L. Gomez \& A. Huiza. 2005. Acantocéfalos del Perú. Rev. Peru. biol. 12: 83-92.

Travassos L. 1917. Contribuicoes para o conhecimento da fauna helmintologica brasileira. VI. Revisao dos acantocefalos brasileiros. Parte 1. Fam. Gigantorhynchidae Hamann, 1892. Mem. Inst. Oswaldo Cruz. 9: 5-62. 
